\title{
NATURAL LANGUAGE INTERFACE TO MOBILE DEVICES
}

\author{
Lina Zhou, Mohammedammar Shaikh and Dongsong Zhang \\ University of Maryland, Baltimore County, Baltimore, MD 21250, U.S.A.
}

\begin{abstract}
Natural language interface (NLI) facilitates the human use of computers. In this paper, we review the state-of-the-art NLI application. Based on the extant literature, we design process flow of an NLI system enabling easy information access via mobile devices.
\end{abstract}

Key words: natural language interface, natural language processing, mobile device

\section{INTRODUCTION}

Natural Language Interface (NLI) is an interface that allows users to interact with the computer using a human language, such as English or Chinese, as opposed to using a computer language, command line interface, or a graphical user interface [1]. NLI essentially provides an abstract layer between users and computers by enabling computers to understand human language instead of the other way around. It allows the user to enter natural language search queries in written or spoken text.

Mobile devices (e.g., PDAs, cell phones) present usability challenges to users and system designers due to small screens and limited input methods, unreliable network connectivity, customer apathy, and other factors [2]. NLI has been used for a variety of applications including database query, question-answering, personalization and so on [3-5]. Despite the potential of NLI in improving the usability of mobile devices, the extant work on NLI has primarily focused on desktop computer systems. Thus, investigating NLI to mobile devices can extend our knowledge on NLI from both theoretical and practical perspectives. 
In this paper, we focus on NLI that enables easy access to information repositories via mobile devices.

\section{NATURAL LANGUAGE INTERFACE TO MOBILE DEVICES}

Natural language processing is the core technology in support NLI systems. A natural language system [6] should not only have an understanding of what a word means and how to combine words into phrases and sentences, but also know what a sentence means in various contexts. The potential of NLI was first demonstrated with LUNAR [7] - a natural language dialogue system. It led to a stream of research on natural language query interfaces to database (e.g., [5]). NLI has been extended to help users navigate the site by accepting and processing questions such as search queries in natural language and responding in natural language [4]. This is particularly useful for transforming customer service capabilities. For example, consumers can issue intuitive and natural commands or queries rather than being asked to follow complex menus and adjust to difficult interfaces. NLI also allows users to control systems by carrying out directions and executing business transactions. The natural language is also viewed as a compelling enabling technology for information personalization [8]. In sum, NLI demonstrates advantages such as efficiency, flexibility, economy, and expressiveness. Speech-based interface can further increase the speed of accessing a computer system by allowing hand-free human computer interaction, which is not possible when using the standard keyboard and mouse.

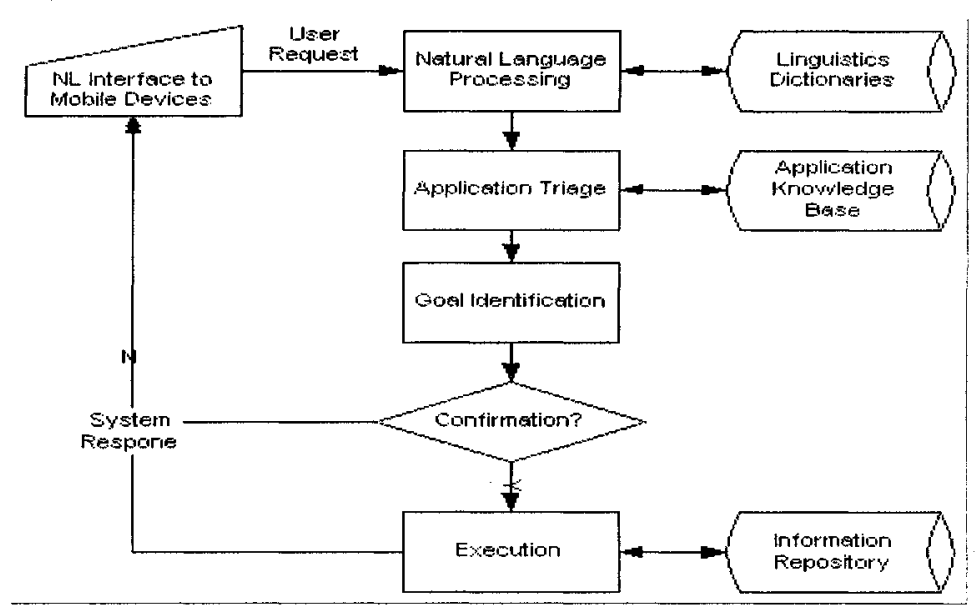

Figure 1. Process Flow of an NLI system for Mobile Devices 
Mobile devices users can benefit from NLI by increasing the efficiency and effectiveness of information management. Mobile devices are identified as the best alternative to paper-based tools in managing information [9]. The physical limitations of mobile devices can be mitigated with the help of natural language. For example, finding an appropriate application and inserting the information into the right place in an application consumes a large amount of users' time. An NLI to applications may help user locate the right application and update information in the corresponding database or information repository.

Drawing from the literature on NLI (e.g., $[10,11]$ ), we design process flow of an NLI system for mobile devices, as shown in Figure 1. Brief descriptions of each component are provided as follows:

NL Interface: The NL interface provides an easy-to-use and unified interface to mobile applications. It accepts text or speech input in natural language.

Natural Language Processing: The user request is processed with natural language techniques.

Application Triage: Based on the understanding result, the user request is triaged to an appropriate application on mobile devices.

Goal Identification and Confirmation: Once the application is identified, a course of action will be initiated by updating information in the application. In addition, confirmation gives users an opportunity to either proceed with the action or return to the NL interface.

Execution: Action is taken in response to user request.

The above processes and related knowledge source and information repository will be updated periodically based on users' interaction history. It is suggested that the challenges of developing satisfactory NLI should never be underestimated [1] and both competence and performance errors should be expected. The effectiveness of the proposed process flow will be evaluated by studying usability of prototype systems in future.

\section{CONCLUSION}

The pragmatic goal of natural language and multimodal interfaces [8] (including speech recognition, keyboard entry, and pointing, among others) is to enable ease-of-use for users/customers in performing more sophisticated human-computer interactions. Information management on mobile devices is an emerging and challenging issue. The NLI presented in 
this paper suggested potential solutions to addressing the above challenges. We hope that this study will motivate more research and applications of NLI to mobile devices.

\section{ACKNOWLEDGEMENTS}

This work is partially supported by the National Science Foundation of USA under Grant\# IIS-0328391. Any opinions, findings and conclusions or recommendations expressed in this material are those of the authors and do not necessarily reflect the views of the National Science Foundation (NSF).

\section{REFERENCES}

1. C. W. Thompson and K. M. Ross, Natural-language interface generating system, in $U . S$. Patent. U.S., 1987.

2. S. Sarker and J.D.W., Understanding Mobile Handheld Device Use and Adoption, Communications of the ACM, vol. 46, pp. 35-40, 2003.

3. J. F. Kelley, An Iterative Design Methodology for User-Friendly Natural Language Information Applications, ACM Transactions on Information Systems, vol. 2, pp. 26 - 41, 1984.

4. E. Paek and H.-j. Jeon, A Natural Language Interface for Simple Classification Tasks, presented at Flairs-97 Preliminary Conference, Gainesville, FL, 1997.

5. A. Yates, O. Etzioni, and D. Weld, A Reliable Natural Language Interface to Household Appliances - EXACT, presented at IUI'03, Miami, FL, 2003.

6. J. Allen, Natural Language Understanding: The Benjamin/Cummings Publishing Company, Inc., 1995.

7. W. Woods, R. Kaplan, and B. Nash-Weber, The Lunar Sciences Natural Language Information System, Bolt Beranek and Newman 2378, June 1972.

8. W. Zadrozny, M. Budzikowska, J. Chai, N. Kambhatla, S. Levesque, and N. Nicolov, Natural language dialogue for personalized interaction, Communications of the $A C M$, vol. 43, pp. $116-120,2000$.

9. C. Campbell and P. Maglio, Supporting Notable Information in Office Work, presented at Conference on Human Factors in Computing Systems, Ft. Lauderdale, FL, 2003.

10.E. Zoltan-Ford, How to Get People to Say and Type What Computers Can Understand, International Journal of Man-Machine Studies, vol. 34, pp. 527-547, 1991.

11. A. Dix, J. Finlay, G. Abowd, and R. Beale, Human-Computer Interaction: Prentice Hall, 1998. 